\title{
Charge Repulsion and Salt-Mediated Charge Screening as Regulators of Amyloid Assembly Pathways
}

\author{
S. E. Hill, T. Miti and Martin Muschol \\ University of South Florida, Tampa, FL,33620
}

Formation of large protein fibrils with a characteristic cross $\beta$-sheet architecture is the key molecular event underlying a wide variety of systemic and neurodegenerative amyloid diseases [1]. Elucidating the mechanisms that regulate amyloid fibril self-assembly is one approach towards developing new drugs and treatment strategies. Recent experiments have strongly implicated oligomeric intermediates, transiently formed during fibril assembly, as critical contributors to cellular toxicity in amyloid diseases. Yet, amyloid fibril assembly can apparently proceed along different assembly pathways that might or might not involve such oligomeric intermediates. Elucidating the mechanisms that determine whether fibril formation involves nonoligomeric or oligomeric pathways, therefore, could have direct implications for development of novel drugs trying to switch assembly from toxic to non-toxic pathways.

We have investigated fibril formation by hen egg white lysozyme, using a combination of static and dynamic light scattering, atomic force microscopy, and circular dichroism [2]. Specifically, we were interested in the question what role, if any the character and the strength of intermolecular protein-protein interactions play in regulating the fibril self-assembly process. Our experiments indicated that amyloidogenic lysozyme switched between three distinct aggregation regimes: initially partially denatured lysozyme formed monomeric linear filaments, which could cross-assemble into higher-order fibers. A second regime was characterized by the formation of globular oligomers, which formed the building blocks for all subsequent fibril assembly. Eventually, this regime gave way to amorphous precipitation. Transitions among these regimes were induced by systematically increasing the ionic strength of the solutions.

We proceeded to characterize the net lysozyme-lysozyme interactions prevailing with the corresponding changes in salt concentration. Intriguingly, fibril assembly only occurred under conditions of net charge repulsion among the amyloidogenic monomers. The transition to net attraction, in turn, coincided with the onset of precipitation. Within the regime of fibril assembly, the transition from monomeric to oligomeric fibril assembly occurred as salt-mediated charge screening become sufficiently effective to begin screening individual charged residues on a single monomer. Based on these observations, we suggest a model of amyloid fibril formation in which repulsive charge interactions become a prerequisite for ordered fibril assembly. We further propose that the spatial extent of salt-mediated screening of charged residues selects between monomeric and oligomeric assembly pathways. It does so by affecting the subset of denatured states that can interact favorably (hydrophobic interactions vs. hydrogen bonding). Charge repulsion also alters the energetic and entropic requirements on the initial intermediates emerging along either the monomeric $v s$. oligomeric assembly paths. Hence, non-specific charge interactions are likely to play a crucial role in selecting amyloid fibril assembly pathways and affecting the structure of the various intermediates emerging along the way [3].

\section{References}

[1] E.H. Koo et al., P.Natl. Acad. Sci USA 96(1999) 9989; C.A. Ross and M.A. Poirier, Nat. Med. 10 (2004) S10; P.T. Landsbury and H.A. Lashuel, Nature 443 (2006) 774.; F. Chiti and C.M. Dobson, Annu. Rev. Biochem. 75 (2006) 333. 
[2] S. E. Hill, et al., (2011) submitted

[3] Acknowledgements: We'd like to acknowledge Dr. Garrett Matthews who generously made his AFM system available to us. Funding for this work was provided, in part, by an NSF IGERT fellowship (S.H.), the Office of Undergraduate Research (T.M.) and grants from the Byrd Alzheimer's Institute (ARG-2007-22) and the BITT Florida Center of Excellence (M.M.)

a) DLS: Nucleation and Growth Kinetics
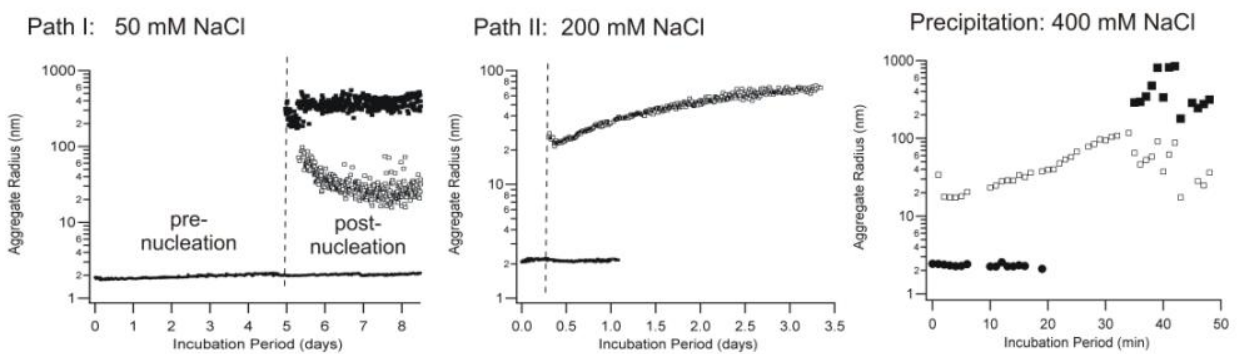

b) AFM: Morphology of Intermediate Aggregates

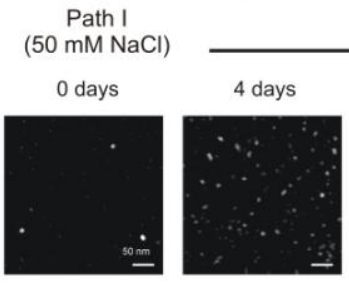
Incubation period

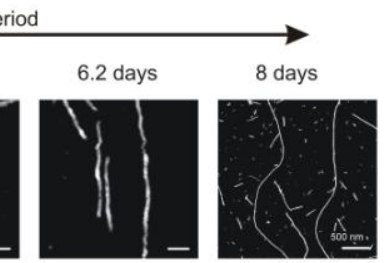

c) AFM: Physical Dimensions of Intermediate Aggregates

pre-nucleation

post-nucleation

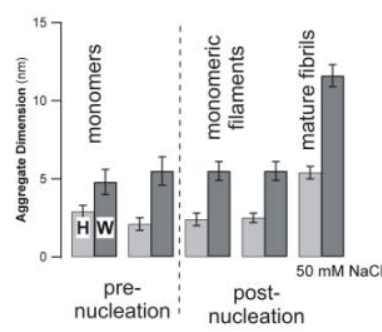

Path II (175 mM NaCl)

$0 \mathrm{hrs}$

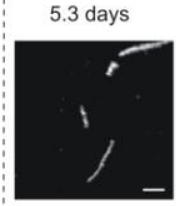

post-nucleation
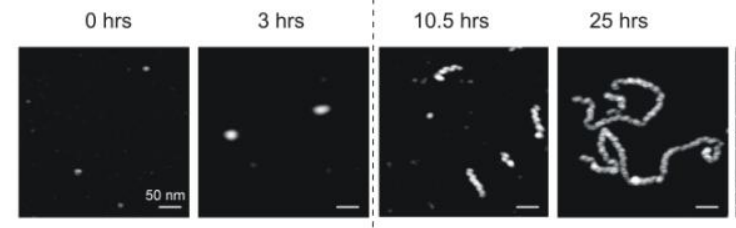

$100 \mathrm{hrs}$

pre-nucleation

post-nucleation

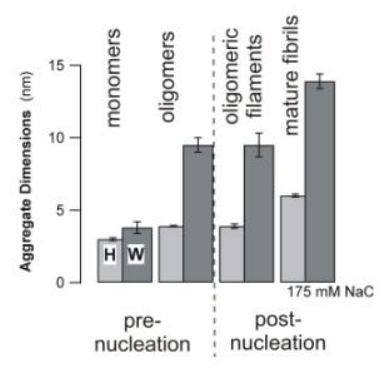

FIG. 1 Aggregation Regimes for Partially Denatured Lysozyme as Function of Salt Concentration.

a) Dynamic light scattering resolves three distinct nucleation and growth regimes for lysozyme samples: At low salt fibril formation causes near-simultaneous nucleation of two nucleus population (Path I). At intermediate salt concentrations, oligomers grow in the lag phase, with nucleation indication formation of protofibrils (Path II). Precipitation sets in at high salt concentrations. b) Morphology and c) Physical dimensions of all intermediates and mature fibrils formed along the non-oligomeric path I and the oligomeric path II obtained from AFM imaging. 